\title{
FUENTES DE LA RESPONSABILIDAD SOCIAL
}

\author{
SOURCES OF SOCIAL RESPONSIBILITY
}

Julio B. Domínguez Granda ${ }^{1}$

\section{INTRODUCCIÓN}

El término "responsabilidad social" (RS), comenzó a utilizarse eN forma generalizada a comienzos de la década del 70 , aunque ya se utilizaba por organizaciones y gobiernos desde tiempos tan antiguos, como finales del siglo XIX; en algunos casos incluso antes. Más adelante, se centró en la empresa, con lo que se generalizo el término "responsabilidad social empresarial" (RSE), o "responsabilidad social corporativa", vinculada a la gran empresa global del mundo desarrollado. En el presente siglo surgió una visión de la responsabilidad social aplicable a diferentes tipos de las organizaciones, denominada entonces responsabilidad social de las organizaciones (RSO), reconociendo que todas ellas tenían responsabilidades en la contribución al desarrollo social.

La fuente de responsabilidad social materia del presente artículo trata de abordar el origen común a todas las acepciones de "responsabilidad social", a fin de contribuir a una adecuada comprensión del término, sustentados en la noción del estado de derecho internacional, con el propósito de promover la toma de conciencia en todo tipo de las organizaciones. A partir de la comprensión de los elementos fuente de la responsabilidad social será posible mejorar la lectura respecto a la RSE y poder generar planes de responsabilidad social en todo tipo de organizaciones.

1 Doctor en Ciencia Política, Rector CEO de la Universidad Católica Los Ángeles de Chimbote, e-mail: rectorado@uladech.edu.pe 
En este sentido, se han identificado elementos fuentes de responsabilidad social. Tales como principios y valores declarados por las empresas u organizaciones, con lo cual claramente se establece un componente ético y social. La filantropía será otro elemento distintivo a ser considerado siempre que esté contenido en la declaración de las estrategias empresariales u organizacionales. La declaración e implementación de elementos del desarrollo sostenible y el cuidado de medio ambiente será crítico para caracterizar un comportamiento socialmente responsable. El concepto de gobernanza es utilizado para desarrollar la idea de que, sin declaraciones de políticas y estrategias no se pueden considerar a las empresas socialmente responsables. Por último, se resalta la importancia del liderazgo para diseñar modelos de educación y formación continua para orientar la formación integral de profesionales con un adecuado componente de formación ciudadana.

\section{LA "RESPONSABILIDAD" Y LO "SOCIAL"}

La noción de responsabilidad desde el punto de vista de la empresa, "tal como se ha usado por los teóricos de la RSE, especialmente en los países desarrollados, obedece a la intención de crear un compromiso corporativo u organizacional que se incorpore a la misión u objetivo de la empresa a través de cada uno de sus diferentes comportamientos y acciones, y que es de naturaleza ética $o$ moral" (Di Filippo, 2012).

Por el lado de la RSO como "voluntad de las organizaciones de incorporar consideraciones sociales y ambientales en su toma de decisiones y de rendir cuenta por los impactos de sus decisiones y actividades en la sociedad y el medio ambiente. Esto implica un comportamiento transparente y ético que contribuya al desarrollo sostenible, cumpla con la legislación aplicable y sea coherente con la normativa internacional de comportamiento" (INDECOPI, 2010). También implica que la responsabilidad social esté integrada en toda la organización, se lleve a la práctica en sus relaciones, y tenga en cuenta los intereses de las partes interesadas.

En tal sentido, se interpreta "responsabilidad" básicamente como compromiso voluntario, éticamente fundado; es decir, no exigible jurídicamente, que corresponde al tema central de la RS y sus diferentes acepciones. Esto concuerda con el Diccionario de la Lengua Española (2007) como "cargo u obligación moral que resulta para uno del posible yerro en una cosa o asunto determinado". 
Etimológicamente alude a la obligación de responder como tema ético planteado de manera perfeccionista en el buen sentido de la palabra. Es ir más allá de las normas morales vigentes y superar las metas o indicadores en que esa norma se concreta.

Lo social en lo referido a la RSE se refiere y aplica al entorno de las personas u organizaciones con intereses directos en el comportamiento de las empresas (trabajadores, clientes, proveedores, sindicatos, accionistas, y otras personas directamente afectadas). Una parte interesada tiene uno o más intereses que puedan verse afectados por las decisiones y actividades de una organización, lo que genera un involucramiento no necesariamente formal o reconocido. A dichas partes interesadas se les denomina stakeholders. En tal sentido, tanto la RSE y la RSO se predican respecto a la organización que la práctica y se aplica a la gestión interna y a sus stakeholders. En tal sentido, lo social se refiere y aplica al entorno de las personas y organizaciones con intereses directos en el desenvolvimiento de la empresa u organización en sus intenciones de lograr desempeños éticamente superiores.

El tema social en las naciones emergentes como el Perú incluye una perspectiva vinculada a las desigualdades por la fuerte concentración en la distribución de la riqueza y del ingreso al igual que en otros países periféricos.

Teniendo en cuenta la literatura sobre el tema de la RSE originada en los países desarrollados de habla inglesa se refiere a la gran firma corporativa y, más específicamente, a la Corporación Transnacional (CT).

En Perú la RSE aplicada a la pequeña y mediana empresa no es significativa, teniendo en cuenta que el $70 \%$ del empleo que se genera proviene de la informalidad.

La visión de que la responsabilidad social es aplicable además a todas las organizaciones (RSO) surgió en la medida que los diferentes tipos de organizaciones, del gobierno, sociedad civil y las pequeñas y medianas empresas, y no solo aquellas grandes empresas vinculadas al mundo desarrollado, tienen responsabilidades en su contribución al desarrollo.

La responsabilidad social RSE/RSO es una contribución activa y voluntaria con ejes sociales, económicos y ambientales, que se integran en el planeamiento estratégico de las organizaciones con el propósito de mejorar su situación, aportar valor a los procesos, beneficiar a los trabajadores y a las partes interesadas dentro del área de influencia. 


\section{FILANTROPÍA Y RESPONSABILIDAD SOCIAL}

$\mathrm{Al}$ reconocer que tanto las organizaciones del ámbito de la RSE como otras organizaciones del alcance de la RSO basan su responsabilidad en el tema ético, estaríamos considerando la responsabilidad social dentro del ámbito del bien común. Esto se explica porque en sus orígenes la responsabilidad social se centraba en las actividades filantrópicas, como, por ejemplo, hacer obras de caridad (Domínguez, 2012).

A nivel académico, principalmente en la educación superior, progresivamente se han venido incorporando diferentes materias a la responsabilidad social; hace más de un siglo aparecen las prácticas laborales y las prácticas justas de operación; actualmente, los derechos humanos, el medio ambiente, la protección del consumidor y la lucha contra el fraude y así otros que llegan a verse como elementos importantes de la responsabilidad social. Pero, ¿se descarta por ello el tema de la filantropía?

Los valores de la verdad, de la justicia y de la libertad, nacen y se desarrollan de la fuente interior de la caridad, que nos hace amar el bien común y nos lleva a buscar efectivamente el bien de todas las personas, consideradas no solo individualmente, sino también en la dimensión social que las une procurando el bien posible para la comunidad en su conjunto. La filantropía que nace del valor de la caridad buscando además que esta se incorpore a programas sociales y/o ambientales llevados a cabo por una empresa u organización. No busca beneficios ni está alineada a la estrategia corporativa, a diferencia de la RSE o la RSO, que busca una inversión socialmente responsable, de allí, por ejemplo, que las empresas alienten a sus trabajadores al voluntariado porque la retención es más alta al mejorar el clima organizacional. De acuerdo a lo anterior, los conceptos de RSE/RSO y la filantropía están estrechamente relacionados como práctica dentro de los aspectos estratégicos de la responsabilidad social en las organizaciones. Es por ello que está relacionada con el "giro del negocio" de la organización y genera un impacto positivo en las comunidades donde opera, al medio ambiente y a la sostenibilidad de la misma. Así, por ejemplo, la universidad podría vincular sus actividades socialmente responsables a la formación integral de los profesionales a los cuales forma vinculándose a la comunidad a través del proceso Extensión Cultural y Proyección Social.

Según lo anterior, las organizaciones alínean sus programas sociales y ambientales al propósito de las mismas. De esta forma los usuarios las percibirán positivamente y no como simple aprovechamiento del tema de la responsa- 
bilidad social haciendo un uso engañoso del término (Green Marketing \& Consulting, 2013).

\section{RESPONSABILIDAD Y BIEN COMÚN}

En la actualidad las condiciones sociales vigentes la vinculan al respeto y a la promoción integral de la persona y sus derechos fundamentales. Tales exigencias atañen, ante todo, al compromiso por la paz, a la salvaguardia del ambiente, a la prestación de servicios esenciales para las personas, algunos de los cuales son, al mismo tiempo, derechos fundamentales del hombre: alimentación, habitación, trabajo, educación, acceso a la cultura, transporte, salud, recreación, teniendo en mente las futuras generaciones.

El bien común es responsabilidad de todos los miembros de la sociedad individual y colectivamente, porque corresponde a una de las inclinaciones más elevadas del hombre; difícil de alcanzar, porque exige la capacidad y la búsqueda constante del bien de los demás como si fuera el propio bien (Conferencia Episcopal Peruana, 2004). La responsabilidad social no se puede desligar del bien común, ya que históricamente se demuestra que la búsqueda exclusiva del interés particular, desligada de consideraciones sobre el bien de la sociedad a la larga da malos resultados (Argandoña, 2011).

Toda forma de organización humana tiene una función social, en clara referencia a las exigencias imprescindibles del bien común; no se puede actuar prescindiendo del efecto del uso de los propios recursos, sino que se debe actuar, además de las ventajas particulares, también las del bien común. En la medida en que las organizaciones, en especial las vinculadas a la sociedad desarrollada se concentren en las ganancias y ampliar su competitividad per se, el bien común pueden verse afectado en términos del desarrollo sustentable y el cuidado del medio ambiente.

\section{DESARROLLO SOSTENIBLE Y CUIDADO DE MEDIO AMBIENTE}

Se refiere a la integración de metas de calidad de vida elevada, la salud, la prosperidad con justicia social: temas sociales que afectan el desempleo, el subempleo, la pobreza y la exclusión social, el mantenimiento de la capacidad de la tierra para conservar la vida en toda su diversidad como vía para expresar las más amplias expectativas de la sociedad en su conjunto atendiendo a la disminución de la desigualdad social, cuidando el entorno natural: agua, suelo, plana y hombre, el espacio exterior y sus interrelaciones. 
"El concepto de Bien Común, como bien comunitario e individual, pero distinto de la mera sumatoria de los bienes individuales, tiene una larga historia que se remonta a 2000 años en el pasado. En el presente parecería haber caído en desuso por el predominio de una noción que prioriza como valor supremo los derechos naturales individuales. Pero el concepto no ha perdido su vigencia en el pensamiento de la Iglesia Católica y se ha reafirmado en la llamada Doctrina Social de la Iglesia, en especial a partir de la Encíclica Rerum Novarum. El Bien Común es la dimensión social y comunitaria del bien moral "el bien de todos los hombres y de todo hombre". Puede desarrollarse una concepción de la Responsabilidad Social Empresaria fundada en el Bien Común y relacionarla con el moderno criterio de responsabilidad hacia los stakeholders. Pero el Bien Común de los stakeholders de una empresa no constituye la totalidad del Bien Común de los stakeholders de toda la sociedad, que es lo implícito en la Doctrina Social de la Iglesia. Y una sociedad que pretenda estar al servicio del ser humano debe proponerse el Bien Común como deber de todos los miembros de la sociedad. Mucho más allá de lo que pretende o aspira la teoría de los stakeholders" (Montuschi, 2009).

La RSE/RSO se ubica equidistante de la propuesta del Milton Frietman, que plantea cuáles deben ser los intereses de las corporaciones que sus agentes deben defender, afirmando que "la responsabilidad social de las empresas es incrementar sus beneficios”; en el otro extremo está el bien común no solo individual sino de toda la colectividad. El enfoque que prevalece es aquel que se refiere al logro del éxito comercial de modo que se respeten los valores éticos, la gente, las comunidades y el medio ambiente.

La responsabilidad de las organizaciones se amplía a todos los stakeholders, a quienes se considera identificados, en cierta medida, con los intereses y negocios de la corporación y a quienes debe responder en una medida que excede lo que establecen los requisitos legales y reglamentarios. Según un último análisis, "no debe verse como la existencia de un conflicto entre el logro del Bien Común y el del bien individual. Se debe considerar que la sociedad es para la persona y que el bien de los individuos no puede contraponerse al de la sociedad de la que forma parte. El bien individual solo puede lograrse dentro de la sociedad y el Bien Común de la sociedad es creado por sus integrantes, realizado por dicha comunidad y compartido con todos sus miembros. Se considera que la organización tiene una obligación hacia todos sus stakeholders por ser la reprehensión de la sociedad de la que forma parte si bien no constituyen la totalidad del Bien Común de los stakeholder de la sociedad. 
Las partes interesadas o stakeholders son organizaciones o individuos que tienen uno o más intereses en cualquiera de las decisiones o actividades de una organización y por este hecho crea una relación con esta, incluso no estando consciente de ello. Una organización puede no estar consciente de todas sus partes interesadas, aunque debería intentar identificarlas. Por su parte, la parte interesada puede que algunas partes interesadas no están conscientes del potencial que tiene alguna organización para afectarla.

El criterio para la identificación de los stakeholders se basa en la existencia de una base real o potencial de una reclamación como la exigencia de un derecho. Pueden estar en el ámbito legal, financiero, o simplemente el derecho a ser escuchado. Quedará más clara su pertinencia cuando se tiene en cuenta su relación al desarrollo sostenible. Por otra parte, la identificación de los impactos de las decisiones o actividades facilitará la identificación de la mayoría de ellas. En tal sentido las organizaciones podrían tener muchas partes interesadas.

Algunas partes interesadas pueden ser internas a la organización como son los empleados que comparten intereses comunes; algunos grupos organizados que apoyan causas sociales podrían ser partes interesadas; otras no están organizadas y podrían ser pasadas por alto o ignoradas.

Algunos elementos que ayudan a identificar a las partes interesadas tendrían que ver con las obligaciones legales de la organización, afectados positiva o negativamente con las decisiones, puedan expresar inquietud por las decisiones, antecedentes de involucramiento, los que puedan ayudar a tratar impactos específicos, afecten la capacidad de la organizaciones para cumplir sus responsabilidades, desfavorecidos por exclusión de involucramientos, afectados en la cadena de valor.

La identificación de las partes interesadas vistas hasta aquí y su involucramiento con la organización son aspectos claves para abordar la responsabilidad social.

El involucramiento con las partes interesadas incluye el diálogo y toma diversas formas a partir de la organización o respuesta de las partes interesadas. Puede tener lugar en reuniones formales o informales; individuales o colectivas. La base es la interacción para ofrecer oportunidades para que las opiniones de las partes interesadas sean escuchadas. De lo que se trata es de conocer las expectativas de las partes interesadas aun cuando estas pueden ser evaluadas por otros mecanismos. El objetivo es que la organización sea consciente del efecto de sus decisiones y actividades sobre los intereses y necesidades de las partes interesadas. 
La responsabilidad social concierne a la totalidad de relaciones de la organización con sus stokeholders visualizándose los siguientes niveles básicos: el primero corresponde a las relaciones con los trabajadores y sobre la organización y calidad del trabajo; en el segundo nivel pueden situarse las responsabilidades que afectan la calidad, la seguridad y utilidad social de los productos o servicios que oferta; constituye el tercer nivel el desarrollo social, conjuntamente con la creación y mantenimiento del empleo y respeto a la legislación de la actividad económica; el cuarto nivel comprende la actitud responsable, la honestidad e integridad en relación a las partes interesadas y el ámbito de la vigilancia de las conductas sociales de los proveedores. A estas cuatro dimensiones se agrega la reivindicación de derechos civiles, sociales y económicos básicos, recogidas en las convenciones de las Naciones Unidas (Perdiguero \& Reche, 2005).

\section{RESPONSABILIDAD SOCIAL Y ESTRATEGIA}

Las actividades vinculadas al desarrollo sostenible y el cuidado de medio ambiente que realizan las organizaciones desde los enfoque de RSE/RSO serán de responsabilidad social cuando formen parte de la estrategia de tales organizaciones. En este sentido la aplicación de la RS tiene que ver con la consecución de objetivos estratégicos y el éxito empresarial u organizacional en el largo plazo al margen de la gestión operativa y del altruismo o cuestiones morales que surjan aleatoriamente y de la gestión operativa. Estas últimas de alguna manera forman parten del planeamiento estratégico al estar presente en los valores corporativos declarados.

La RS puede ofrecer numerosos beneficios potenciales para una organización como sigue: toma de decisiones en base a la compresión de las expectativas de la sociedad y el riesgo de ignorarla, gestión de riesgos, gestión de la innovación, mejora competitiva, mejora de su relación con las partes interesadas, mejora del clima organizacional, mejora de la salud y seguridad en el trabajo, mejora de la motivación y retención del personal, ahorros por mejora de productividad, entre otros.

La gobernanza de la organización es el factor más importante que hace posible que una organización se responsabilice de los impactos de las decisiones o actividades y para integrar la responsabilidad social en toda la organización y sus relaciones con lo cual valida esta función. 


\section{GOBERNANZA}

La gobernanza de la organización es el sistema por el cual se toman las decisiones estratégicas para lograr objetivos.

Se basa en la estructura y procesos definidos así como la influencia de la cultura y los valores en la organización que conforman el marco de toma de decisiones. Está conformada por el gobierno y la estrategia de quien tiene la autoridad y responsabilidad de perseguir los objetivos de la organización. Por tanto se diferencia de la gestión del día a día.

La gobernanza, además, es un medio para aumentar la capacidad de las organizaciones de comportarse de manera socialmente responsable respecto a las estrategias de responsabilidad social que incorpore en su planeamiento estratégico. Esto es así porque permite a la organización supervisar y poner en práctica los principios de responsabilidad social inherente a las organizaciones también conocidos como principios de gobernanza de las organizaciones.

El diseño del planeamiento estratégico debería basarse en comportamientos y actitudes que estén de acuerdo con los principios aceptados de una buena gobernanza en el contexto de situaciones específicas teniendo en cuenta la diversidad social, ambiental, legal, cultural, política y organizativa. Dicho principios son los siguientes:

\section{PRINCIPIOS DE GOBERNANZA DE LAS ORGANIZACIONES}

Rendición de cuentas: implica que la dirección de la organización responde al control de la alta dirección y que la organización a su vez responde ante las autoridades competentes en cuanto a las leyes y regulaciones.

En cuanto a la RS debe responder frente al desarrollo sostenible y el medio ambiente, ante los afectados por su actuar así como ante la sociedad en general. Incluye la responsabilidad cuando se cometen errores, acciones correctivas para repararlos y preventivas para que no se repitan.

Transparencia: las organizaciones ponen a disposición de modo accesible sobre las políticas, decisiones y actividades de forma clara precisa y completa para facilitar que las partes interesadas evalúen con exactitud el impacto de las decisiones y actividades en relación a sus propios intereses.

Comportamiento ético: el comportamiento de una organización debe ser ético es decir basado en valores de la honestidad, equidad e integridad lo que implica preocupación por personas, animales y medio ambiente y el compromiso de 
tratar el impacto de sus decisiones respecto a los intereses de partes interesadas. Para ello debe declarar sus valores y principios fundamentales; utilización de una estructura de gobierno que ayude a promover comportamientos éticos; adopción de normas de comportamiento ético; resolución de conflictos que se presenten; mecanismos de supervisión y revisión del comportamiento ético entre otras.

Respeto a los intereses de las partes interesadas: consiste en el respeto de los derechos, reclamaciones e intereses de las partes interesadas. Para lo cual cada organización debe identificar a las partes interesadas evaluando su capacidad para contactar, involucrarse e influir en la organización.

Respeto al principio de legalidad: la organización reconoce la supremacía del derecho y a la idea de que ningún individuo u organización está por encima de la ley. En el contexto de responsabilidad social, implica que una organización cumple con las leyes y las regulaciones aplicables. Lo que implica conocer los requisitos legales y reglamentarios e informar la necesidad de su cumplimiento e implementación dentro de la organización.

Respeto a la normativa internacional de comportamiento: la organización reconoce como parte del principio de legalidad el derecho internacional consuetudinario, principios de derecho internacional generalmente aceptados o 0 acuerdos, tratados o convenciones intergubernamentales, reconocidos de manera universal o casi universal. La normativa internacional de comportamiento en relación a la salvaguarda ambiental o social debe respetarse preferentemente.

Respeto a los derechos humanos: la organización debería respetar los derechos humanos y reconocer, tanto su importancia como su universalidad. Incluye la promoción y difusión de la Carta Universal de los Derechos Humanos.

Fuente: Op Cit. ISO 26000 p.17-23.

Por su parte, los responsables del gobierno de una organización deben diseñarla de tal manera que formalicen una estructura jerárquica y determinen y apliquen procesos a través de los cuales fluya la aplicación de los principios descritos como parte del cumplimiento de los requisitos legales y reglamentarios formalmente, o incorporados a la cultura y valores organizacionales. La implementación del sistema de gestión en base a la estructura y procesos permite aplicar los principios y prácticas de responsabilidad social.

El siguiente es el perfil de una estructura y procesos en una organización para permitir aplicar principios y prácticas de responsabilidad social: 


\begin{tabular}{|c|c|}
\hline $\begin{array}{c}\text { Característica de la estructura y procesos } \\
\text { en el planeamiento estratégico }\end{array}$ & Aporte a la responsabilidad social \\
\hline $\begin{array}{l}\text { Desarrolla estrategias, objetivos y metas que } \\
\text { incluyen RS. }\end{array}$ & Reflejan el compromiso hacia la RS. \\
\hline Indicador de rendición de cuentas. & Demuestra compromiso por parte de los líderes. \\
\hline Indicador de cultura organizacional. & $\begin{array}{l}\text { Crear y nutrir un ambiente y cultura en los que } \\
\text { se practiquen los principios de la RS. }\end{array}$ \\
\hline Indicador de motivación e incentivos. & Motivación e incentivos asociados a la RS. \\
\hline Indicadores de eficacia. & $\begin{array}{l}\text { Usa eficientemente los recursos financieros } \\
\text { naturales y humanos. }\end{array}$ \\
\hline Prácticas justas para las promociones. & Promueve oportunidades justas. \\
\hline Identificación de partes interesadas. & $\begin{array}{l}\text { Equilibra las necesidades de la organización } \\
\text { con los de las partes interesadas. }\end{array}$ \\
\hline Indicador de sistema de comunicación. & $\begin{array}{l}\text { Procesos de comunicación en dos direcciones } \\
\text { con las partes interesadas. }\end{array}$ \\
\hline Indicador de participación de trabajadores. & $\begin{array}{l}\text { Promueve la participación de trabajadores en } \\
\text { actividades de RS. }\end{array}$ \\
\hline Eficacia de la coordinación interna. & $\begin{array}{l}\text { Equilibra el nivel de autoridad, responsabilidad } \\
\text { y capacidad de las personas que toman deci- } \\
\text { siones. }\end{array}$ \\
\hline Documentar y registrar los procesos. & $\begin{array}{l}\text { Determina la rendición de cuentas por los resul- } \\
\text { tados de las decisiones. }\end{array}$ \\
\hline Sistema de gestión de la calidad. & $\begin{array}{l}\text { Revisa y evalúa periódicamente los procesos } \\
\text { de gobernanza de la organización, establecer } \\
\text { mejoras y comunicar los cambios. }\end{array}$ \\
\hline
\end{tabular}

Fuente: Op Cit. ISO 26000 p. xii.

\section{INDUCIR LA RESPONSABILIDAD SOCIAL A TRAVÉS DE LA FORMACIÓN DE CAPITAL HUMANO}

Desde la RS se propone introducir grados crecientes de eficiencia y de integridad moral en los seres humanos que dirigen y controlan las organizaciones sin desconocer que este trabajo no es suficiente por estar articulado a las responsabilidades de otros responsables del campo tecno económico y social implicados por velar por los temas sociales.

El liderazgo es clave en la gobernanza de una organización eficaz. Esto es 
cierto no solo para la toma de decisiones, sino también para motivar a los colaboradores a que practiquen la responsabilidad social y para integrar a través de la cultura de la organización.

La instalación de la responsabilidad social en el pensamiento de los líderes de las organizaciones depende de la generación de estímulos necesarios y suficientes para que las organizaciones avancen en tal sentido. Uno de los ejes al respecto, demostrado por la crisis de corrupción en los últimos 20 años, se relaciona con la endeble formación ética de las personas que ocupan posiciones claves en el desarrollo social. En tal sentido, se requiere cambios en la educación y la formación de personas, de tal manera de generar nuevos hábitos y costumbres relacionadas con la madurez, compresión y entrega. Estos no responden a imposiciones externas ni a los contenidos de asignaturas. La ética ejercita la reflexión moral estando la RS alineada a una forma de gestión que surge alineada a dicha práctica. De allí que la cuestión central respecto a la incorporación de la RS como estrategia sea la educación. El problema está relacionado con la formación de líderes que "construyan organizaciones responsables con sus empleados, respetuosas con sus consumidores, promuevan la preservación del medio ambiente, se involucre en los grandes problemas de interés colectivo; y que a la vez no tengan doble código de ética" (Barbieri, 2012).

Cuando se habla de RS se hace referencia a valores, a un modelo educativo integrador y transversal que tiene en cuenta no solo las competencia técnica, sino también los valores humanos aplicados a ellos. Dentro de las organizaciones igualmente se refiere a un modelo de formación continua con las mismas característica. Este modelo supone el aprendizaje para toda la vida, adquirir valores, conocimientos y competencias que permitan dar soluciones a problemas sociales, económicos y medioambientales. En tal sentido, el currículo incluirá los valores del desarrollo sostenible, ética profesional, impacto de las tecnologías y procesos en términos de sostenibilidad social y ambiental, consumo y comportamiento responsable de la comunidad, política alimentaria, la organización del transporte, el tratamiento de residuos, la eficiencia energética, la calidad del empleo, o las actividades de voluntariado, cooperación y acción social entre otros. En la misma línea, los programas de formación continua que consideran el impacto ambiental de las actividades económicas, la gestión responsable de los recursos naturales, la política de igualdad, la calidad el trabajo, en dialogo social entre otras.

Para que las instituciones educativas y las organizaciones puedan hacer frente con éxito a esos nuevos desafíos será necesario introducir mejoras en la 
gobernanza y adoptar modelos de calidad que incluyan los principios, valores y prácticas del desarrollo sostenible dentro de los proyectos estratégicos.

Por su parte la Norma Internacional de Responsabilidad Social de las Organizaciones, ISO 26000, además de los principios descritos anteriormente, presenta un conjunto de materias fundamentales y asuntos a considerar en programas de RS en la educación y la formación continua en las organizaciones como sigue:

- Derechos humanos: debida diligencia, situaciones de riesgo, complicidad, reclamaciones, discriminación, derechos civiles y políticos, derechos económicos sociales y culturales, principios del trabajo.

- Prácticas laborales: trabajo y relaciones laborales, condiciones de trabajo, diálogo social, salud y seguridad en el trabajo, desarrollo humano y formación en el lugar de trabajo.

- El medio ambiente: contaminación, uso sostenible de los recursos, mitigación y adaptación al cambio climático, protección del medio ambiente.

- Prácticas justas de operación: anticorrupción, participación política responsable, competencia justa, promoción de la RS en la cadena de valor, respeto al derecho de propiedad.

- Asuntos de consumidores: prácticas justas de marketing, información objetiva y prácticas justas de contratación.

- Protección de la salud y la seguridad de los consumidores, consumo sostenible, servicios de atención al cliente, apoyo a la resolución de quejas y controversias, protección de la privacidad de datos, acceso a servicios esenciales, educación y toma de conciencia.

- Participación activa y desarrollo de la comunidad: Participación activa de la comunidad, educación y cultura, creación de empleo y desarrollo de actividades, desarrollo y acceso a la tecnología, generación de riqueza e ingresos, salud, inversión social.

\section{CONCLUSIONES}

Las fuentes de la RS se identifican como los elementos comunes básicos de las diferentes acepciones del término, como son la Responsabilidad Social Empresarial, Responsabilidad Social Corporativa o Responsabilidad Social de las Organizaciones, entre otros. 
La función de la responsabilidad social es aplicable a empresas u organizaciones formales del gobierno, la sociedad civil y las empresas respecto a aspectos internos, como el clima laboral y, externos, como el interés estratégico en relación al desarrollo sostenible y el medio ambiente.

Siendo una función estratégica debe ser diseñado, implementado y difundido en los planes estratégicos de mediano plazo e incluso en la planificación de largo plazo.

Implica necesariamente el diseño de estructuras y procesos articulados con sistemas de gestión de calidad y de información y comunicación integradas.

La cultura organizativa debería estar gestionada aplicando instrumentos validados que permitan medir el cambio de las actitudes y valores de personal de la organización así como de sus usuarios. Dicho sistema debe tener en cuenta los principios y valores corporativos declarados.

La eficacia de la gobernanza o modelo de gobierno sobre el cual se toman las decisiones es requisito para las políticas de responsabilidad social se interrelacionen y complementen; una gobernanza con tal característica permite a una organización tomar acciones respecto a las estrategias consideradas para el comportamiento socialmente responsable.

Históricamente se demuestra que la búsqueda exclusiva del interés particular alejada de los intereses del bien de la sociedad la expone a malos resultados por lo que la responsabilidad social debe tener implícita y explícitamente el bien común.

\section{REFERENCIAS}

ARgandoñA, A. (2011). El bien común. Documento de Investigación DI-937. Bussines Scholl Universidad de Navarra. Recuperado el 24 de octubre de 2014, de http://scholar.google.es/ scholar?start $=10 \& q=$ bien + comun + en + la + empresa\&hl $=$ es\&as_sdt $=0,5$

BARBIERE, A. (2012). El discernimiento ético como umbral de los modelos de enseñanza en investigación en Iberoamérica. En I. Licha, Enseñanza de la Responsabilidad Social Empresarial: Retos de las universidades en Iberoamérica (págs. 63-71). Buenos Aires, Argentina.

Conferencia Episcopal Peruana, Pontificio Consejo de "Justicia y PaZ". (2004) Compendio de la doctrina social de la Iglesia. Lima, Perú: EPICONSA.

Di FILIPPO, A. (2012). La Responsabilidad Social Empresarial (RSE) como instrumento de vinculación universidad-sociedad, En I. Licha, Enseñanza de la Responsabilidad Social Empresarial: Retos de las universidades en Iberoamérica (págs. 25-62). Buenos Aires, Argentina.

DomíngueZ, J. (2012). Conceptualización sobre la responsabilidad social específica de una universidad católica, En J. Domínguez, C. Rama, La Responsabilidad Social Universitaria en la Educación a Distancia (págs. 53-74). Chimbote, Perú: ULADECH Católica. 
Green Marketing \& Consulting (18 de noviembre de 2013). Filantropía Empresarial y Responsabilidad Social Corporativa ¿Cuál es la diferencia? Recuperado el 23 de octubre de 2014, de http://b-green.pe/filantropia-empresarial-y-responsabilidad-social-corporativa-cual-es-ladiferencia/

INDECOPI. (2010) Norma Técnica Peruana, Guía de Responsabilidad Social, NTP-ISO 26 000. Lima, Perú.

MontusCHI, L (2007). El Bien Común, la Responsabilidad Social Empresarial y el pensamiento social de la Iglesia. Recuperado el 23 de octubre de 2014, de https://ideas.repec.org/p/cem/ doctra/363.html

PERDiguero, T \& ReCHE, G. (2005). Responsabilidad social de las empresas y los nuevos desafios de la gestión empresarial. Recuperado el 24 de octubre de 2014, de http://books.google.es/books? $\mathrm{hl}=\mathrm{es} \& \mathrm{lr}=\& \mathrm{id}=\mathrm{aUJ} c j h 0 \mathrm{ov} 8 \mathrm{C} \& \mathrm{oi}=$ fnd\&pg $=\mathrm{PA} \& \& \mathrm{dq}=$ responsabilidad + en $+\mathrm{la}+$ empresas + \&ots $=$ wrYKcIZjYH\&sig = AsH1rqmZ-nnzRObcUwL4Th_1i4\#v=onepage\&q= responsabilidad $\% 20 \mathrm{en} \% 20 \mathrm{la} \% 20 \mathrm{empresas} \& \mathrm{f}=$ false 